\title{
Polybacterial stimulation suggests discrete IL-6/IL-6R signaling in human fetal membranes: Potential implications on IL-6 bioactivity
}

\author{
Nathalia Mayumi Noda-Nicolau ${ }^{\mathrm{a}, \mathrm{b}}$, Jossimara Polettini ${ }^{\mathrm{a}, \mathrm{b}, \mathrm{c}}$, Márcia Guimarães da Silva ${ }^{\mathrm{b}}$, \\ Morgan R. Peltier ${ }^{\mathrm{d}, \mathrm{e}}$, Ramkumar Menon ${ }^{\mathrm{a}, *}$

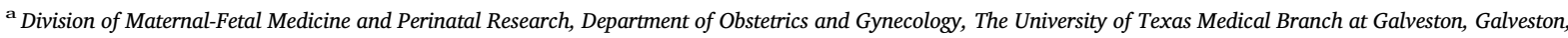 \\ TX, United States \\ ${ }^{\mathbf{b}}$ Department of Pathology, Botucatu Medical School, UNESP - Univ. Estadual Paulista, Botucatu, São Paulo, Brazil \\ ${ }^{\mathrm{c}}$ Master's Course in Health Sciences, University of Western São Paulo, UNOESTE, Presidente Prudente, São Paulo, Brazil \\ ${ }^{\mathrm{d}}$ Department of Biomedical Research, NYU-Winthrop University Hospital, Mineola, NY, United States \\ e Department of Obstetrics and Gynecology, NYU-Winthrop University Hospital, Mineola, NY, United States
}

\section{A R T I C L E I N F O}

\section{Keywords:}

Fetal membranes in vitro

Genital mycoplasmas

Interleukin-6

Polybacterial infection

\begin{abstract}
A B S T R A C T
The polybacterial invasion of the amniotic cavity and risk of preterm birth is often due to cervicovaginal bacteria such as genital mycoplasmas (Mycoplasma hominis and Ureaplasma urealyticum) and Gardnerella vaginalis. The most studied biomarker associated with preterm birth is interleukin-6 (IL-6), a pleiotropic cytokine that performs different functions based on classical or trans-signaling mechanisms. This study evaluated the changes in IL-6 and IL- 6 function associated accessory molecules by human fetal membranes to determine the functional availability of IL-6 assessment in an in vitro model of polybacterial infection. Fetal membranes were treated with LPS or heat-inactivated genital mycoplasmas and G. vaginalis alone or in combination. IL-6 and its soluble receptors (sgp130, sIL-6R) were assessed in conditioned medium by immunoassays and membrane-bound receptors were evaluated in the tissue using immunohistochemistry and RT-PCR. Data from protein and gene expression were evaluated using linear mixed effects models. Data from immunohistochemistry were evaluated using one-way analysis of variance followed by the Tukey test. Genital mycoplasmas alone, or in combination, inhibited IL-6 trans-signaling with increased sgp130 production. G. vaginalis activated the classical IL-6 signaling pathway, as did LPS. Polybacterial treatment resulted in a balanced response with neither pathway being favored. The increase in IL-6 production by fetal membranes in response to infection is likely a non-specific innate response and not an indicator of a functional mediator of any labor-inducing pathways. This suggests that correlating the risk of adverse pregnancy outcomes and designing interventions based on IL- 6 levels without considering soluble receptors may be an ineffective strategy.
\end{abstract}

\section{Introduction}

Intraamniotic infection and inflammation are associated with spontaneous preterm birth (PTB) (Goldenberg et al., 2000; Helmo et al., 2017). These infections are generally due to cervicovaginal colonizers (DiGiulio, 2012; Mendz et al., 2013) such as genital mycoplasmas (Mycoplasma hominis and Ureaplasma urealyticum) and Gardnerella vaginalis (Menard et al., 2010), a pathogen frequently associated with bacterial vaginosis (Santos-Greatti et al., 2016) that ascends from the lower genital tract. M. hominis and $U$. urealyticum are the most commonly isolated bacteria in the amniotic fluid in both PTB with intact membranes and in preterm premature rupture of membranes (pPROM)
(DiGiulio, 2012). Both species, along with G. vaginalis, are present at higher frequencies in human fetal membranes from women with preterm labor (Doyle et al., 2014). Although the relative roles of bacterial diversity, bacterial load and the host response to infection in the induction of PTB remain unclear, polybacterial invasion of the amniotic cavity (PIAC) is thought to trigger an inflammatory response in fetal membranes (Romero et al., 2006), which, in turn, can increase prostaglandin production and lead to preterm contractions that result in preterm labor and ultimately preterm delivery.

Inflammation by microbial pathogens is heterogeneous, and there are no predictive biomarkers for predicting PTB. In a recent study published by our group (Noda-Nicolau et al., 2016), different

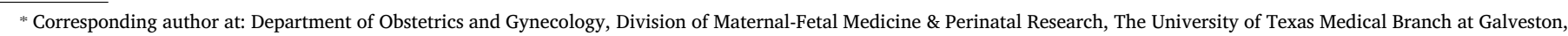
301 University Blvd, MRB 11-158, Galveston, TX 77555, United States.

E-mail address: ram.menon@utmb.edu (R. Menon). 

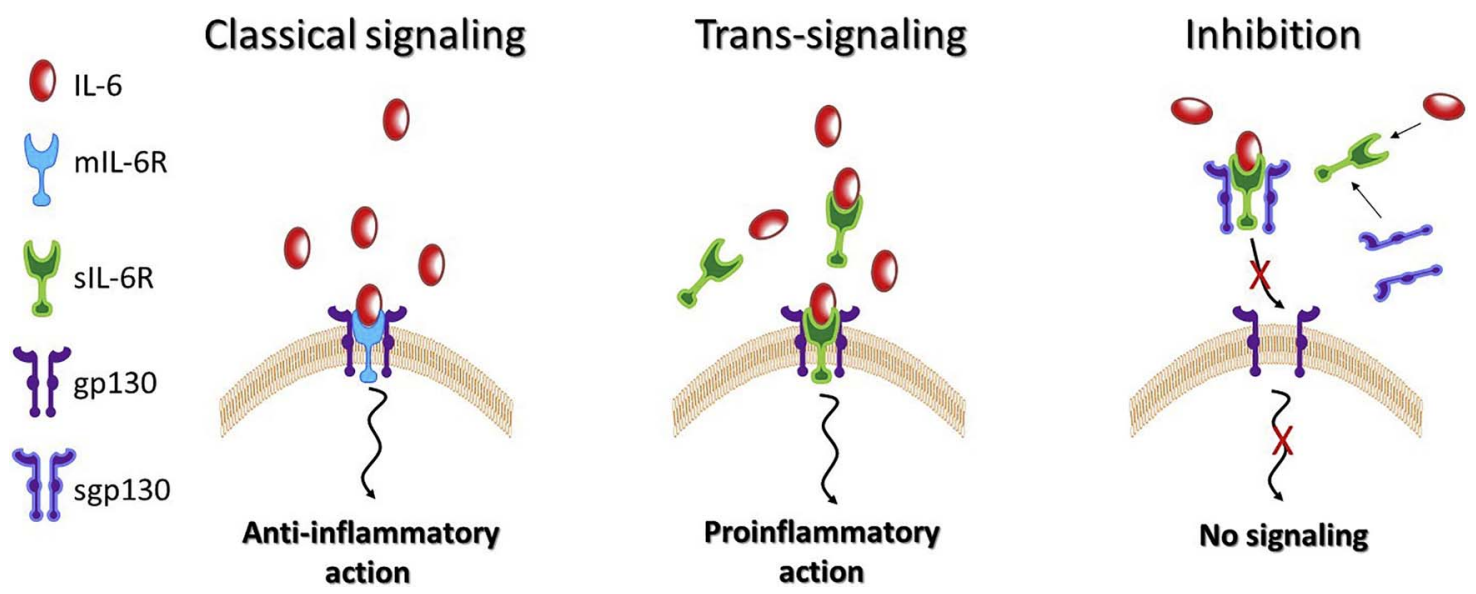

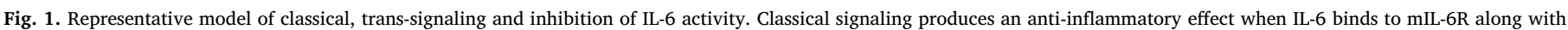

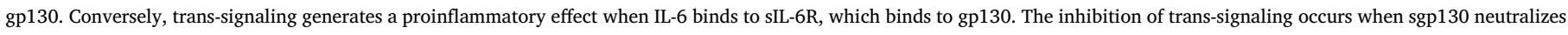

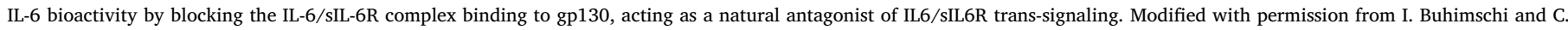
Buhimschi ${ }^{13}$

combinations and loads of bacterial species had distinct effects on the array of cytokines produced in membrane host immune response. Therefore, using a single inflammatory biomarker to evaluate the health of asymptomatic pregnancies may not be feasible.

The most studied biomarker associated with PTB is interleukin- 6 (IL-6) (Menon et al., 2011). High levels of IL-6 in cervicovaginal fluid (Taylor et al., 2013) and amniotic fluid (Kim et al., 2013) are associated with infection and PTB. IL-6 is a pleiotropic cytokine that performs its functions either through classical or trans-signaling mechanisms, which determine its biological activities (Fig. 1). Classical signaling, through the membrane-bound IL-6 receptor (mIL-6R), produces an anti-inflammatory effect; conversely, trans-signaling through the soluble IL- 6 receptor (sIL-6R) generates a proinflammatory effect. In both cases, the binding between IL-6 and mIL-6R or sIL-6R is followed by homodimerization of $\beta$-subunit glycoprotein 130 (gp130), the signal transduction subunit. However, soluble gp130 (sgp130) functions as a natural antagonist of IL6/sIL6R trans-signaling, neutralizing IL-6 bioactivity by blocking the IL-6/sIL-6R complex binding to gp130 (Lee et al., 2011; Rose-John, 2012) and suppressing inflammation. To better understand how polybacterial infections may affect IL- 6 signaling and bioactivity, we evaluated the expression of IL- 6 and its various IL- 6 receptors (mIL-6R and sIL-6R) and signaling modifiers (gp130 and sgp130) in conditioned medium and tissues from a polybacterial infection tissue culture model of fetal membranes.

\section{Materials and methods}

\subsection{Patient and collection of fetal membranes}

Fetal membranes were obtained from elective repeat cesarean sections at term ( $>37$ weeks of gestation) prior to the onset of labor $(n=6)$. The decidua and adhering blot clots were removed from the membranes using saline and sterile cotton gauze. Segments $(6 \mathrm{~mm}$ disks isolated with a skin biopsy punch), were placed in Hanks balanced salt solution (HBSS; Sigma Chemical Co., St. Louis, MO, USA) containing penicillin/streptomycin $(10 \mathrm{IU} / \mathrm{mL})$ and amphotericin $\mathrm{B}(1 \mathrm{IU} / \mathrm{mL})$ and incubated at $37^{\circ} \mathrm{C}, 5 \% \mathrm{CO}_{2}$ for $48 \mathrm{~h}$. Culture medium was replaced every $24 \mathrm{~h}$, as previously described (Fortunato et al., 1994; Menon et al., 2009).

Women with a prior history of pPROM or spontaneous PTB were excluded, as were patients with obstetrical and medical complications and infections (subclinical infections as indicated by high C-reactive protein levels, foul smelling vaginal discharge, fever, and those undergoing antibiotic treatment). The Institutional Review Board at The
University of Texas Medical Branch (UTMB), John Sealy Hospital, Galveston, TX, approved this study on October 7, 2011 until April 15, 2016 (IRB \#: 11-251).

\subsection{Stimulation of fetal membranes}

The preparation of heat-killed stocks of $U$. urealyticum (ATCC \#27816), M. hominis (ATCC \#14027) and G. vaginalis (ATCC \#49145) is described in our previous report (Noda-Nicolau et al., 2016). Considering that the usual load used in the most of in vitro studies is $10^{6}$ CFU(Flores-Herrera et al., 2012; Noda-Nicolau et al., 2016; ZagaClavellina et al., 2007), we employed this load and $10^{3} \mathrm{CFU}$ (an arbitrary value) of heat-inactivated bacteria to stimulated tissue cultures for $24 \mathrm{~h}$ performing different treatments as decribed in Table 1 . The negative control consisted of sterile tissue medium, and purified lipopolysaccharide (LPS) from Escherichia coli (O55:B5) at $100 \mathrm{ng} / \mathrm{mL}$ was used as a positive control. Supernatants and tissues were then harvested and stored at $-80^{\circ} \mathrm{C}$ until biochemical analysis, as described below. Additional tissue samples were fixed in $10 \%$ formalin for paraffin embedding and immunohistochemistry.

\subsection{Protein biomarker assays}

A customized cytokine panel (EMD, Millipore, Billerica, MA, USA) based on Luminex xMAP ${ }^{\mathrm{TM}}$ technology (Luminex, Austin TX) was used to quantify IL-6 and sIL-6R concentrations in conditioned medium. A commercially available ELISA kit (R\&D Systems, Minneapolis, MN, USA) was used to quantify sgp130 levels in these samples. All assays were performed according to the manufacturer's instructions. Samples in which cytokine levels were estimated to be below the sensitivity of the assay were set equal to the sensitivity of the assay and those with

Table 1

Treatments used to stimulate fetal membranes in vitro.

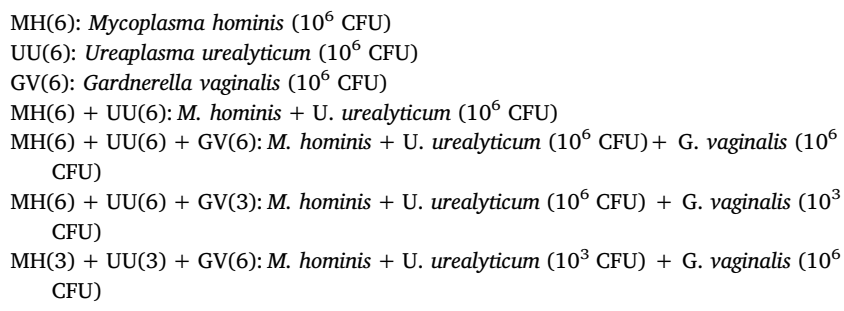

$\mathrm{CFU}=$ colony forming unit. 

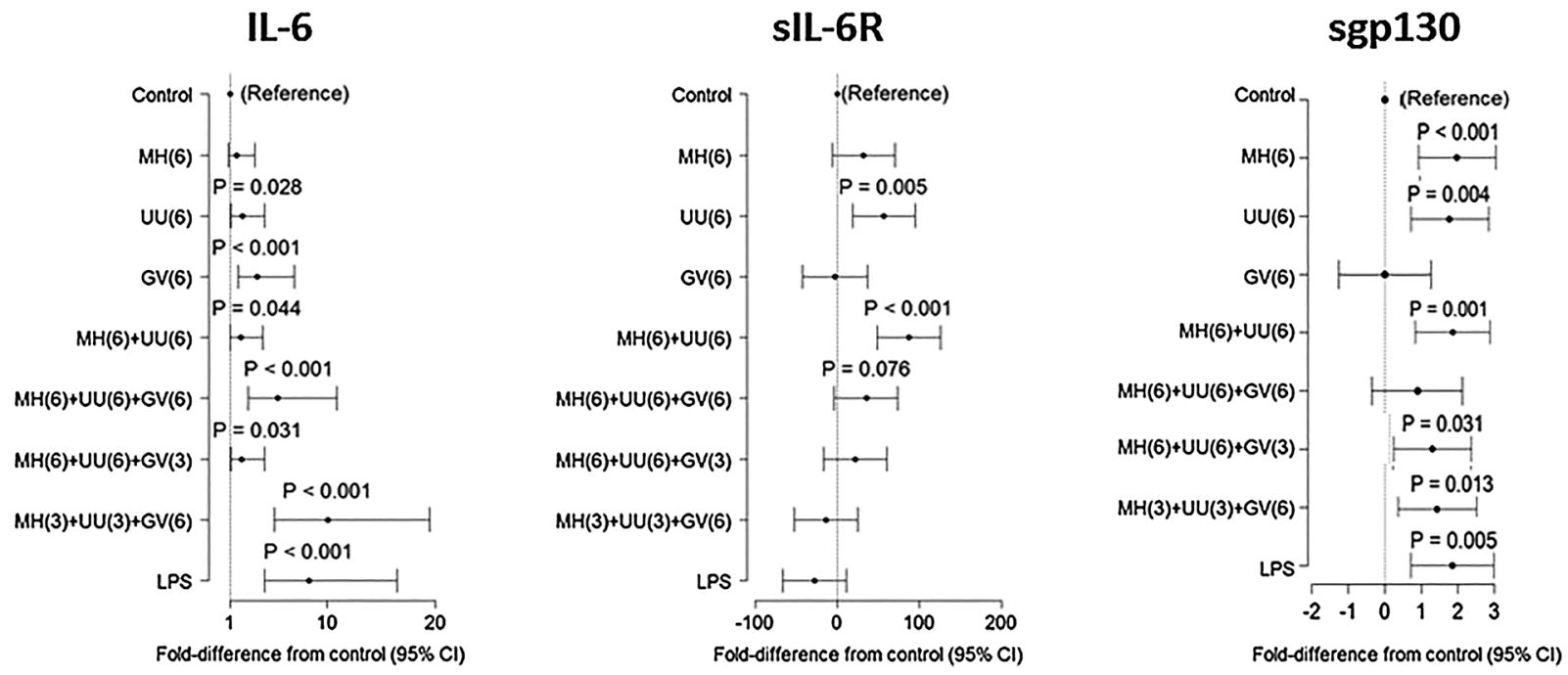

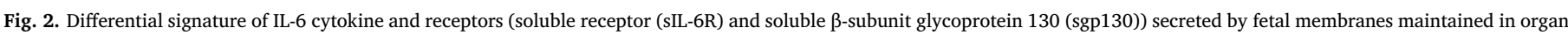

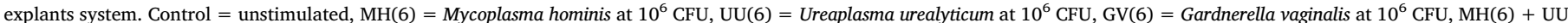

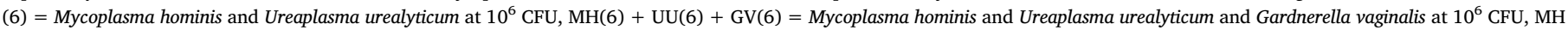

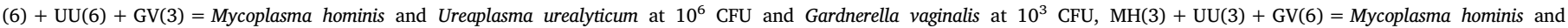
Ureaplasma urealyticum at $10^{3} \mathrm{CFU}$ and Gardnerella vaginalis at $10^{6} \mathrm{CFU}$, LPS-lipopolysaccharide.

concentrations at levels above standard curve were diluted and re-assayed.

\subsection{Gene expression}

Frozen fetal membranes were homogenized in $1 \mathrm{~mL}$ of Trizol (Invitrogen, Grand Island, NY, USA), and extracted using Direct-zol RNA Mini Prep kits (Zymo-Research, Irvine, CA, USA), according to the manufacturer's instructions. The quality and concentration of extracted total RNA were measured by UV-spectroscopy. Aliquots of the RNA samples $(0.1 \mathrm{mg} / \mathrm{mL})$ were then reverse transcribed using the FirstStrand cDNA Synthesis SuperScript II reverse transcriptase kit (Invitrogen, Carlsbad, CA, USA). Relative mRNA expression levels of gp130 (hs00174360 m1) and mIL-6R (hs00169842 m1) and the housekeeping gene, GAPDH, were measured using TaqMan Gene Expression Assay (Applied Biosystems, Foster City, CA, USA). The absence of DNA contamination was confirmed by amplifying mock controls of RNA that were not reverse transcribed.

\subsection{Immunohistochemistry analysis}

Fetal membranes embedded in paraffin blocks were sectioned $(6 \mu \mathrm{m}$ thick) and subject to deparaffinization, rehydration and antigen retrieval by boiling in Trilogy ${ }^{\mathrm{TM}}$ solution (Cell Marque, Hot Springs, AR, USA) for $30 \mathrm{~min}$. Sections were then treated with Peroxidazed $1^{\mathrm{TM}}$ (Biocare Medical, Pacheco, CA, USA) and Protein block ${ }^{\mathrm{TM}}$ (Spring Bioscience, Pleasanton, CA, USA) followed by Mach $4^{\mathrm{TM}}$ Universal HRP Polymer Kit with DAB (Biocare Medical, Pacheco, CA, USA). The primary antibodies used were: rabbit anti-human mIL-6R (sc-661, dilution 1:150, $3 \mathrm{~h}$ of incubation at $4^{\circ} \mathrm{C}$, Santa Cruz Biotechnology, Dallas, TX, USA) and mouse anti-human gp130 (sc-9994, dilution 1:25, overnight incubation at $4{ }^{\circ} \mathrm{C}$, Santa Cruz Biotechnology, Dallas, TX, USA). Sections were counterstained with Harris hematoxylin. In this system, a brown color indicates positive staining for immunolocalization in the slide. Images from three different areas from each slide were analyzed using Image $\mathrm{J}$ software and applying hematoxylin-diaminobenzidine (H-DAB) specific color deconvolution in the RBG (red-green-blue) image of the amnion and chorion layers. Median intensity was calculated using the measure function. Since the maximum intensity value of RGB image analyzed in the program is 255 , and the amount of chromogen present is inversely proportional the intensity value, in order to facilitate the interpretation of results, we deducted the value of 255 from median intensities represents in the graph, making the results directly proportional.

\subsection{Statistical analysis}

Data from protein and gene expression were evaluated using linear mixed effects models using the lme4 library of the R statistical language. Effects due to tissue donor were considered random and all other effects were fixed. Differences between individual treatments were ascertained using contrast procedures and results are expressed as differences from control with 95\% confidence intervals (CI). The residuals of all fit models were analyzed for compliance with the assumptions of parametric statistical techniques (normality, independence and equality of errors). Parametric bootstrapping was performed (on 10,000 replicates) and data were reanalyzed whenever violations of these assumptions were detected. Immunohistochemistry results were evaluated using one-way analysis of variance (ANOVA) followed by Tukey's test with GraphPad Prism, in which a p value $<0.05$ was considered to be statistically significant.

\section{Results}

The combination of different doses of genital mycoplasmas and $G$. vaginalis are defined here as polybacterial treatments, and the results of these treatments, were compared with cultures from unstimulated samples (negative control).

G. vaginalis, alone or in combination with genital mycoplasmas, significantly increased IL-6 production. Although other treatment groups, except for $M$. hominis, also showed increased IL- 6 concentrations (Fig. 2), the increase was not as great as that produced by $G$. vaginalis.

Soluble IL-6R concentrations did not change in response to polybacterial treatment, LPS, M. hominis or G. vaginalis. However, U. urealyticum significantly increased sIL-6R production alone, or in combined treatment with $M$. hominis (Fig. 2). In addition, the sgp130 concentration was increased by LPS as well as M. hominis and U. urealyticum, and in the polybacterial treatments, except for the combination of the same load of all the studied bacteria. G. vaginalis also had no effect on sgp130 production (Fig. 2). The increase in IL-6, sIL-6R and sgp130 during genital mycoplasma stimulation indicates the binding of 


\section{mIL-6R}

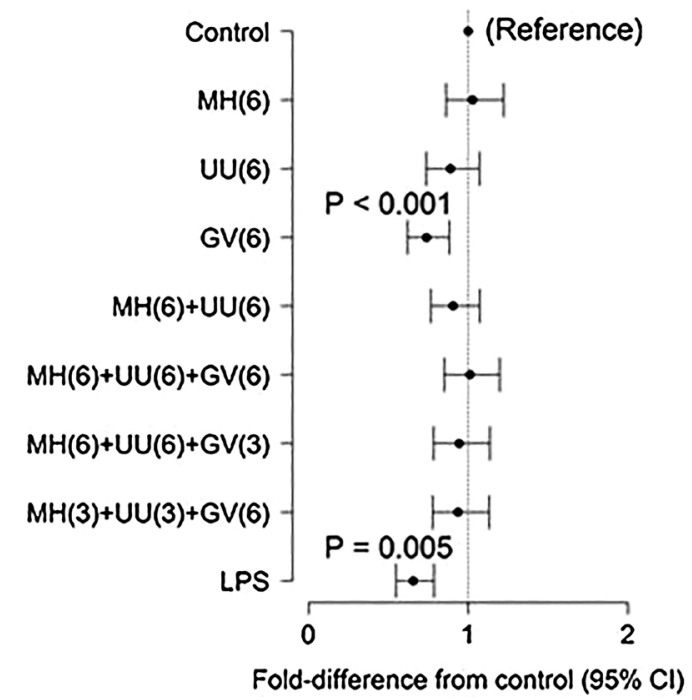

\section{gp130}

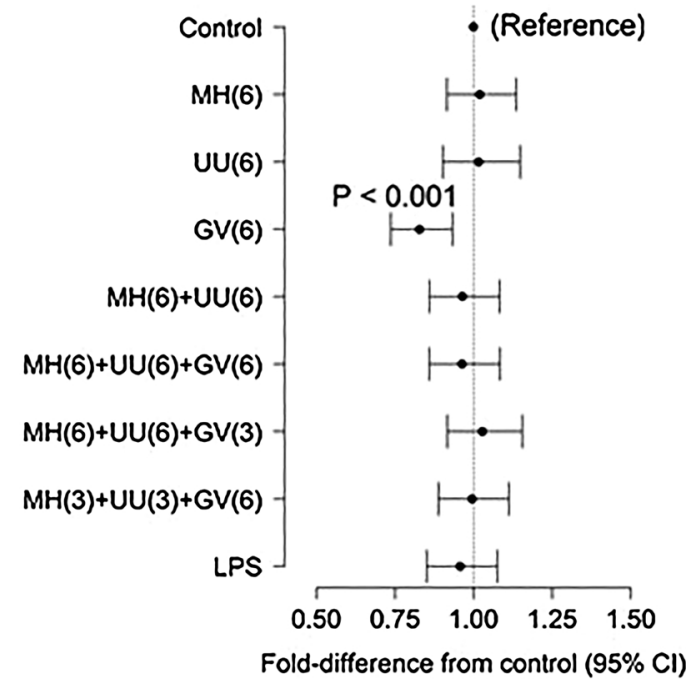

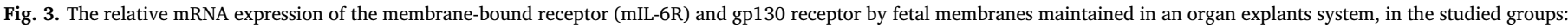

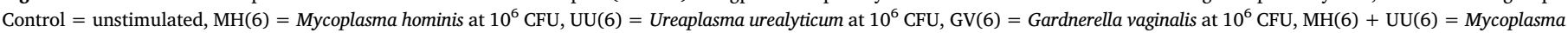

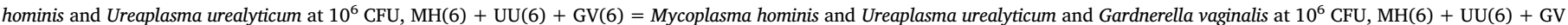

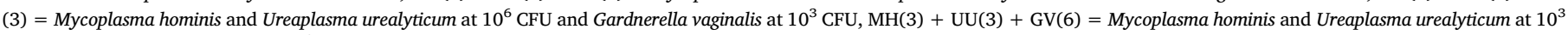
CFU and Gardnerella vaginalis at $10^{6} \mathrm{CFU}$, LPS = lipopolysaccharide.

these mediators; thus, there was no interaction with membrane-bound receptors, thereby inhibiting the proinflammatory action of IL- 6 (transsignaling). Similarly, in the polybacterial stimulation with different loads of bacteria, sgp130 was increased but without sIL-6R, suggesting the inhibition of trans-signaling.

The mRNA levels of membrane-bound receptors, i.e. mIL-6R and gp130, were reduced by $G$. vaginalis, but LPS was only effective at reducing mIL-6R expression. No other effects of bacterial stimulation were detected (Fig. 3). Immunohistochemistry analysis suggested that neither polybacterial stimulation nor treatment with $M$. hominis or $U$. urealyticum affected mIL-6R levels in amnion or chorion cells (Fig. 4), and only LPS and $G$. vaginalis treatments increased mIL-6R expression in amnion cells (Fig. 4; p < 0.001). Moreover, gp130 was not changed following treatment with genital mycoplasmas, but it was increased in both amnion and chorion cells by LPS, G. vaginalis and all polybacterial treatments (Fig. 5; p < 0.001), suggesting the activation of classical signaling in the presence of $G$. vaginalis or LPS.

\section{Discussion}

Polybacterial infections are commonly associated with spontaneous PTB and pPROM (DiGiulio, 2012; DiGiulio et al., 2010; Marconi et al., 2011). However, the host inflammatory response varies with the bacterial load, the combination of bacteria, and the tissues affected (Menon et al., 2009; Noda-Nicolau et al., 2016; Zaga-Clavellina et al., 2007). This non-homogenous immune profile complicates the search for biomarkers of intrauterine infections and the development of interventions (Menon et al., 2007; Ryckman et al., 2009). Genital mycoplasmas are one of the most common isolates from pregnancies with intra-amniotic infection (IAI) or PIAC (DiGiulio, 2012). However, recent findings question the contribution of genital mycoplasmas to adverse outcomes (Choi et al., 2012; Govender et al., 2009; Lee et al., 2009). Our prior work (Noda-Nicolau et al., 2016), showed that genital mycoplasmas are not highly immunogenic in fetal tissues; in fact, they tend to buffer overwhelming inflammation generated by other pathogens by increasing the anti-inflammatory cytokine response (increasing IL-10, IL13 and sTNFR2). In this study, we further tested the role of polybacterial infections on the innate immune response by fetal membranes. Since IL- 6 is one of the most studied cytokines as a marker of IAI and inflammation (Menon et al., 2011) and it is used clinically in multiple point of care (POC) test kits to diagnose these conditions (Chaemsaithong et al., 2015a,b), we studied how the host response may modulate IL- 6 and its signaling molecules during PIAC.

In Fig. 6, our data were used to build a representative model of classical, trans-signaling and inhibition of IL- 6 activity after $24 \mathrm{~h}$ of fetal membrane treatment. In summary, we observed that: 1) our positive control (LPS) activated the IL-6 classical signaling pathway, since the expression of membrane-bound receptors was increased, along with IL6. 2) G. vaginalis activated IL-6 classical signaling, since IL-6 was increased along with gp130 and mIL-6R, similar to the LPS response. Of note, the effect of LPS and G. vaginalis treatment induced an anti-inflammatory response, although they both increased IL-6 significantly compared to the control treatment. 3) $M$. hominis has no detectible effect on altering either IL- 6 or IL- 6 receptor levels; therefore, $M$. hominis is unlikely to have any effect on IL-6 signaling. 4) The increase in IL-6 and sIL-6R expression by $U$. urealyticum alone or in combination with M. hominis (genital mycoplasmas) suggests that an upregulation of these molecules needed for trans-signaling activation; however, the concurrent increase in sgp130, which inhibits trans-signaling, suggests that this response is either balanced or tightly regulated. Classical signaling is also unlikely with Mycoplasma stimulation, as membrane bound-receptors were not increased upon exposure to these pathogens. 5) Polybacterial stimulation with similar or different loads of pathogens increased IL- 6 and gp130 levels, but had no effect on mIL-6R. This suggests an impairment of the biological activity of IL-6 via classical signaling. Similarly, no changes in sIL-6R were observed in response to polybacterial treatment, which suggests that any effect was triggered by trans-signaling. Finally, polybacterial treatment did not show potential for an IL-6-mediated effect, as all markers tested showed a balanced response regardless of treatment (combination and load). Our results confirm that the type of bacteria and bacterial load play major roles in determining IL- 6 production, the expression of its receptors, and likely its bioactivity.

The high prevalence of vaginal, cervical and intra-amniotic 


\section{A) mIL-6R}
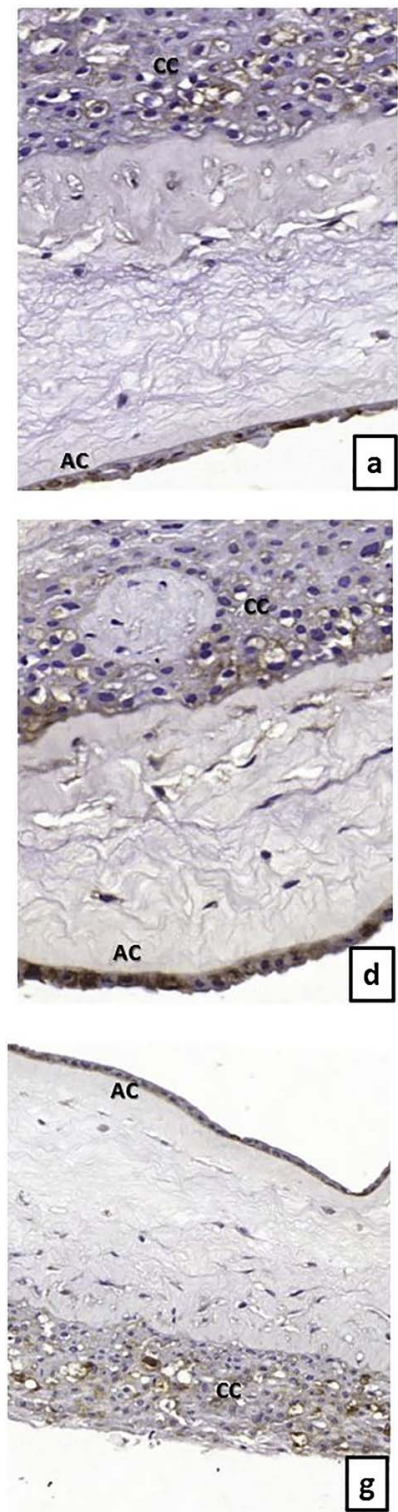

B)

mIL-6R in amnion cells

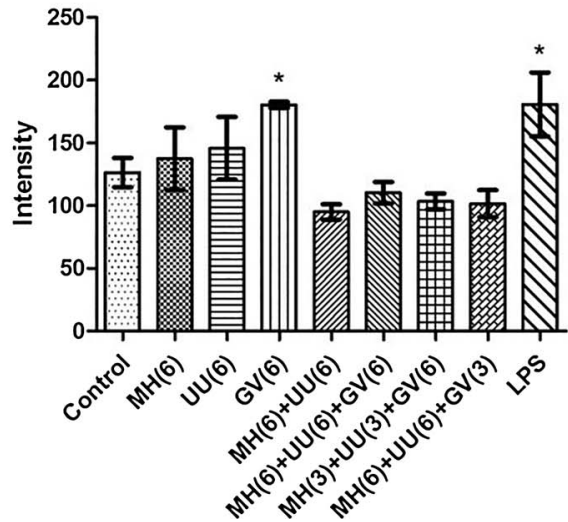

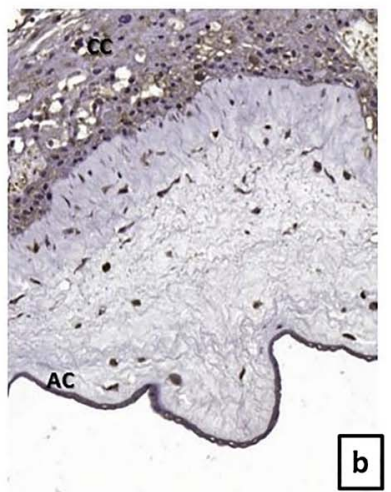
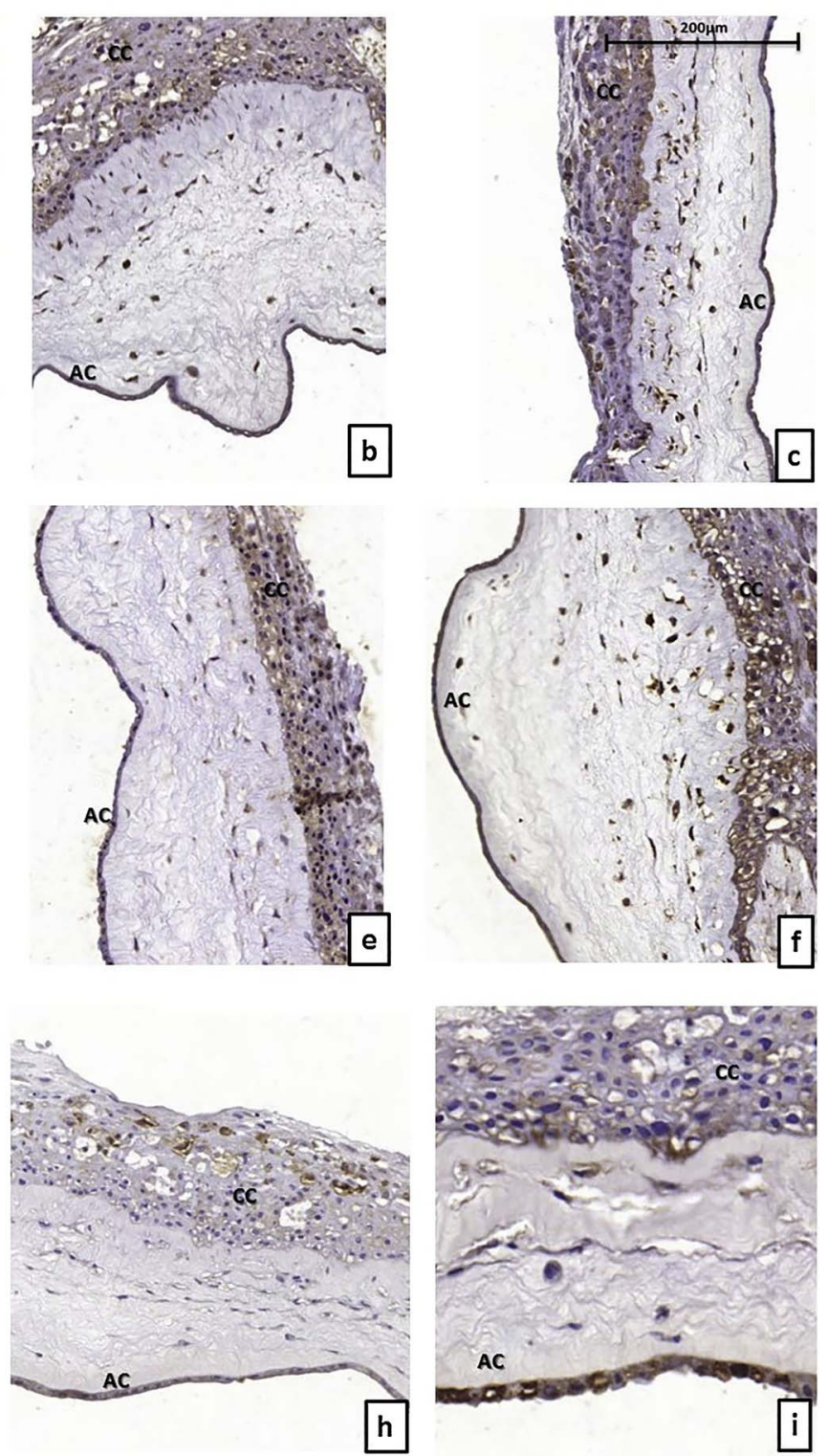

Fig. 4. A) Immunohistochemical staining for $\mathrm{mIL}$ $6 \mathrm{R}$ in the amnion (ac) and chorion (cc) maintained in an organ explants system for each treatment: a) Control = unstimulated, b) $\mathrm{MH}(6)=$ Mycoplasma hominis at $10^{6} \mathrm{CFU}$, c) $\mathrm{UU}(6)=$ Ureaplasma $u r$ ealyticum at $10^{6} \mathrm{CFU}$, d) $\mathrm{GV}(6)=$ Gardnerella vaginalis at $10^{6} \mathrm{CFU}$, e) $\mathrm{MH}(6)+\mathrm{UU}(6)=$ Mycoplasma hominis and Ureaplasma urealyticum at $\left.10^{6} \mathrm{CFU}, \mathrm{f}\right)$ $\mathrm{MH}(6)+\mathrm{UU}(6)+\mathrm{GV}(6)=$ Mycoplasma hominis and Ureaplasma urealyticum and Gardnerella vaginalis at $10^{6} \mathrm{CFU}$, g) $\mathrm{MH}(6)+\mathrm{UU}(6)+\mathrm{GV}$ (3) = Mycoplasma hominis and Ureaplasma urealyticum at $10^{6} \mathrm{CFU}$ and Gardnerella vaginalis at $10^{3}$ $\mathrm{CFU}$, h) $\mathrm{MH}(3)+\mathrm{UU}(3)+\mathrm{GV}(6)=$ Mycoplasma hominis and Ureaplasma urealyticum at $10^{3} \mathrm{CFU}$ and Gardnerella vaginalis at $10^{6} \mathrm{CFU}$, i) LPS = lipopolysaccharide. B) Differential intensity of gp130 in amnion cells by immunohistochemistry in the studied treatments compared to control. * $\mathrm{p}<0.001$. C) Differential intensity of gp130 in chorion cells by immunohistochemistry in the studied treatments compared to control. colonization by genital mycoplasmas have been extensively demonstrated in pregnancy complications, such as PTB and pPROM (Capoccia et al., 2013; Kacerovský et al., 2009; Kwak et al., 2014). Additional studies have shown that IAI is associated with mycoplasmas and increased IL- 6 concentrations in the amniotic fluid (Romero et al., 2014; Yoon et al., 2003). These observations have suggested that IL-6 may be a valuable biomarker, and resulted in the development of POC tests (Chaemsaithong et al., 2015a,b; Musilova et al., 2016) designed 


\section{A) GP130}
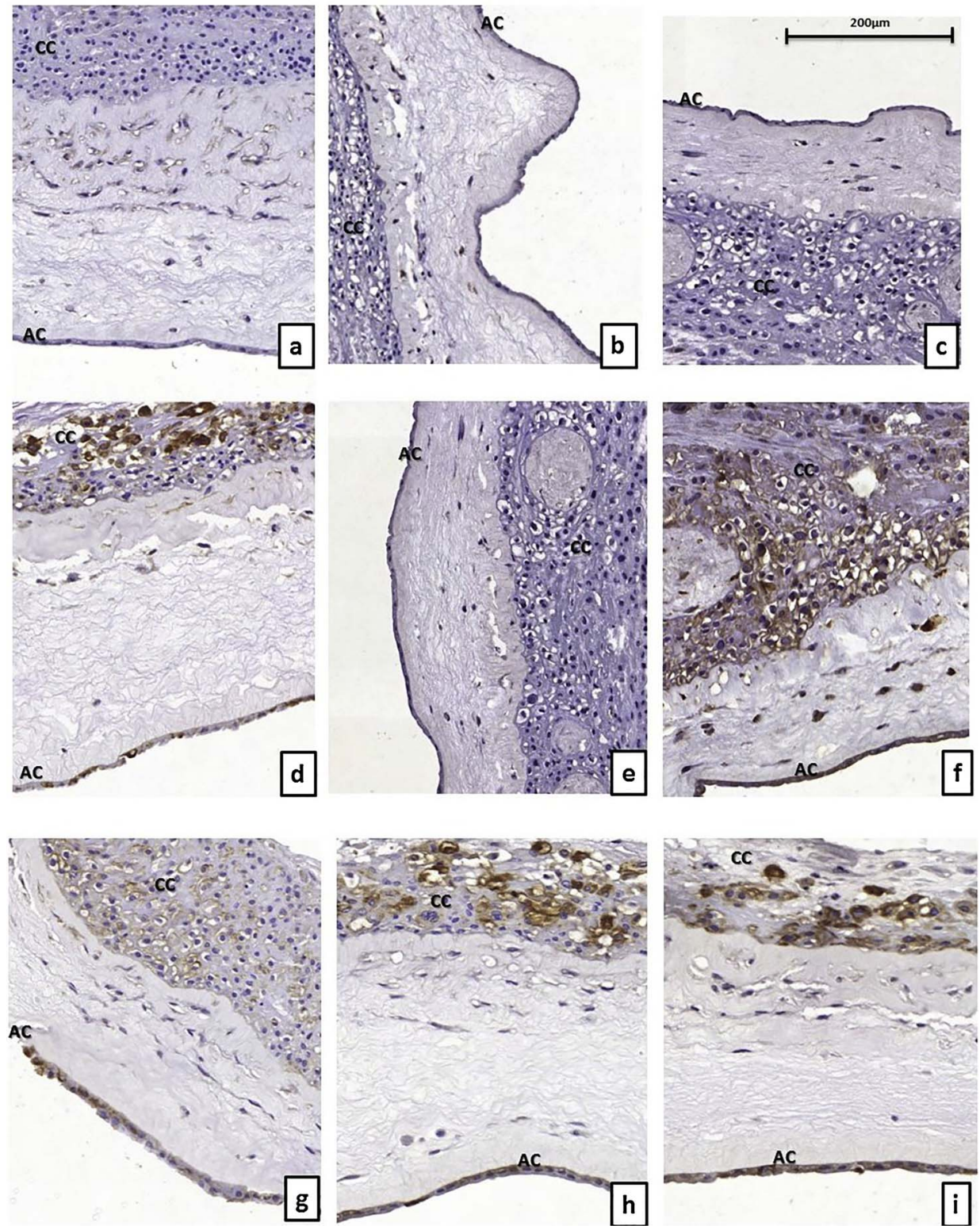

B) gp130 in amnion cells

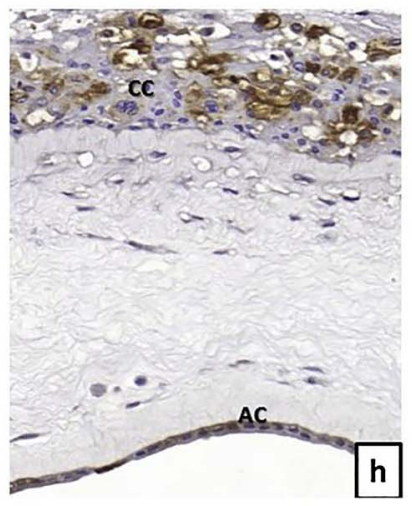

c)
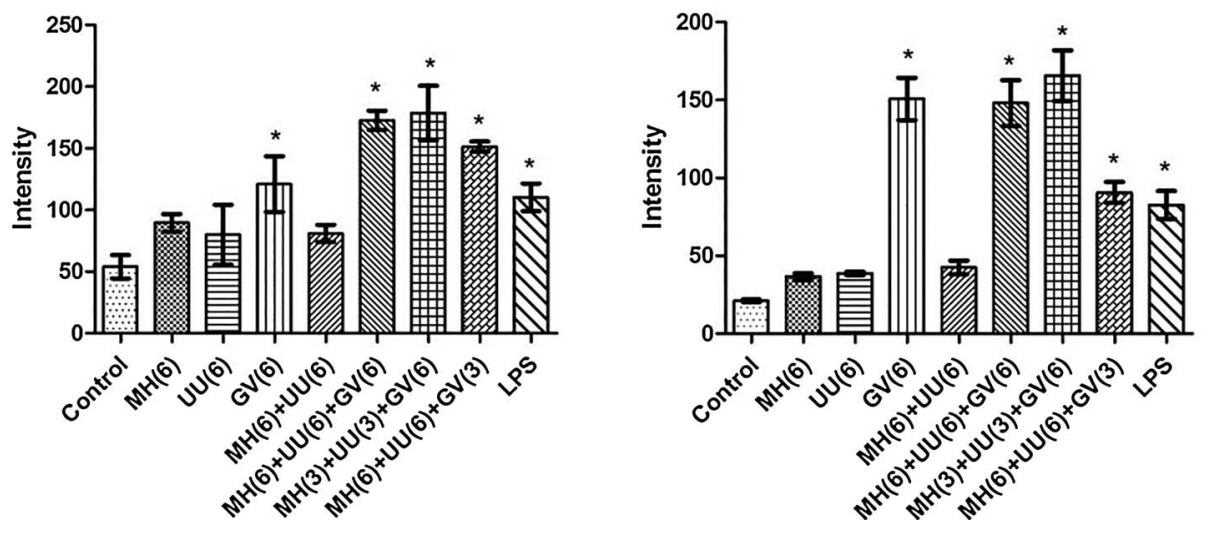

Fig. 5. A) Immunohistochemical staining for gp130 in the amnion (ac) and chorion (cc) maintained in an organ explants system for each treatment: a) Control = unstimulated, b) $\mathrm{MH}(6)=$ Mycoplasma hominis at $\left.10^{6} \mathrm{CFU}, \mathrm{c}\right) \mathrm{UU}(6)=$ Ureaplasma urealyticum at $10^{6} \mathrm{CFU}$, d) $\mathrm{GV}(6)=$ Gardnerella vaginalis at $10^{6} \mathrm{CFU}$, e) $\mathrm{MH}(6)+\mathrm{UU}(6)=$ Mycoplasma hominis and Ureaplasma urealyticum at $\left.10^{6} \mathrm{CFU}, \mathrm{f}\right) \mathrm{MH}(6)+\mathrm{UU}$ (6) $+\mathrm{GV}(6)=$ Mycoplasma hominis and Ureaplasma urealyticum and Gardnerella vaginalis at $\left.10^{6} \mathrm{CFU}, \mathrm{g}\right) \mathrm{MH}$ (6) $+\mathrm{UU}(6)+\mathrm{GV}(3)=$ Mycoplasma hominis and Ureaplasma urealyticum at $10^{6} \mathrm{CFU}$ and Gardnerella vaginalis at $10^{3} \mathrm{CFU}$, h) $\mathrm{MH}(3)+\mathrm{UU}(3)+\mathrm{GV}$ (6) = Mycoplasma hominis and Ureaplasma urealyticum at $10^{3} \mathrm{CFU}$ and Gardnerella vaginalis at $10^{6} \mathrm{CFU}$, i) LPS = lipopolysaccharide. B) Differential intensity of gp130 in amnion cells by immunohistochemistry in the studied treatments compared to control. ANOVA followed by Tukey's test, * $\mathrm{p}<0.0001$. C) Differential intensity of gp130 in chorion cells by immunohistochemistry in the studied treatments compared to control. ANOVA followed by Tukey's test, * $\mathrm{p}<0.001$ for clinical use. However, information on quantitative change alone is not sufficient to understand the functional downstream effects of IL-6 that activate or inhibit labor pathways. Therefore, we tested changes in other molecules that IL-6 interacts with to exert its biological effects in response to polybacterial pathogens associated with PTB. It is possible that increased circulating sgp130 levels is part of a convalescent response to minimize IL-6 production hours after the initial exposure. Quantification of sIL-6R and sgp130 may also be useful for identifying 
N.M. Noda-Nicolau et al.

Journal of Reproductive Immunology 126 (2018) 60-68

UPS

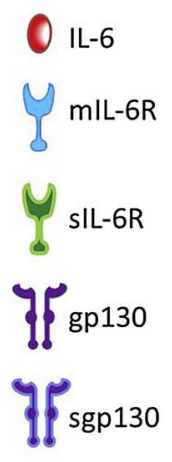

Gardnerella vaginalis
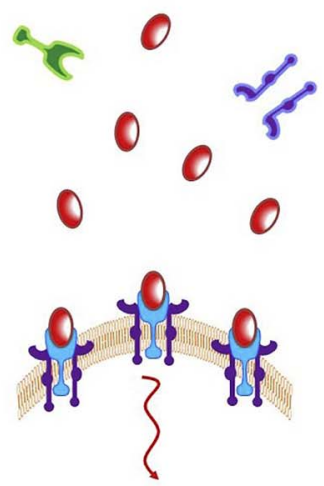

Anti-inflammatory action

Mycoplasma hominis Ureaplasma urealyticum Genital mycoplasmas

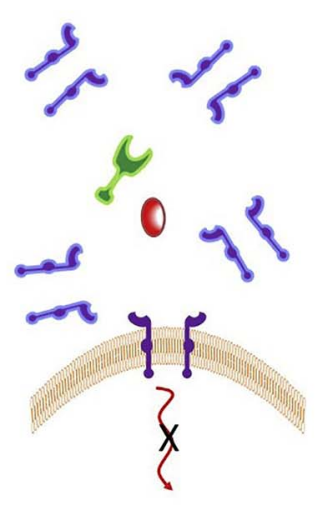

No signaling

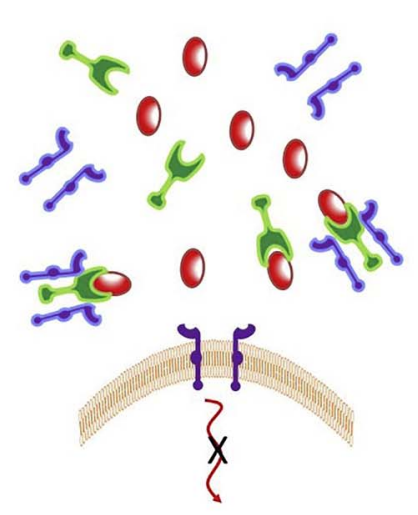

No signaling

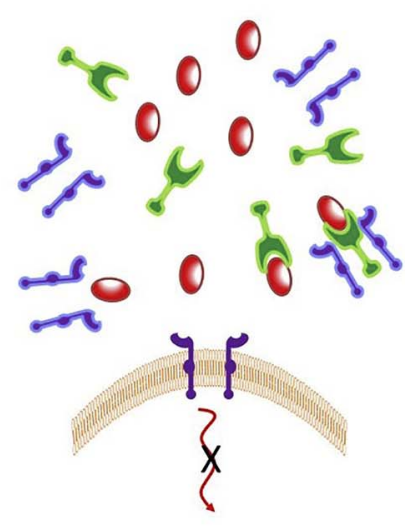

No signaling
Polybacterial stimulation with same loads

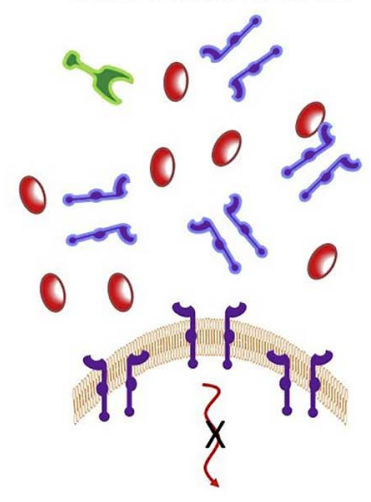

No signaling
Polybacterial stimulation with different loads

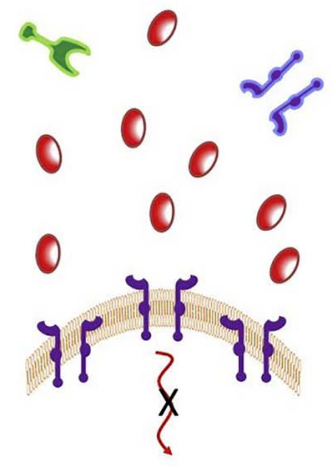

No signaling

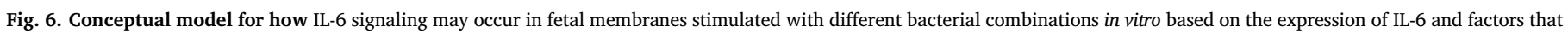
affect its biological activities.

66 
patients most likely to benefit from sgp130Fc therapy due to excessive IL-6 trans-signaling (Jones et al., 2011). Our results regarding the inhibition of IL- 6 trans-signaling through increased sgp130 could also help explain why many pregnancies that test positive for genital mycoplasmas and other microorganisms do not result an adverse pregnancy outcomes (Aagaard et al., 2014; Govender et al., 2009; Lee et al., 2009; Naessens et al., 1989; Steel et al., 2005). Inhibition of IL-6 transsignaling results in the downregulation of inflammatory signaling, which could also favor the survival of Ureaplasma species that live off the urea-rich (Shepard and Lunceford, 1967) amniotic fluid and need to evade the maternal immune system. Similarly, polybacterial stimulation with different loads of genital mycoplasma and $G$. vaginalis do not suggest a functional role for IL- 6 through either the classical or transsignaling pathways. Therefore, although IL- 6 may increase in quantity in response to polybacterial infection, the presence of genital mycoplasmas may mitigate the function of IL- 6 as an anti-inflammatory mediator. A disturbance or immune weakness in the uterine cavity may attract mycoplasma due to an underlying pathology, forcing them to ascend from the genital tract. Based on data from this report and our prior report (Noda-Nicolau et al., 2016), we postulate that this ascension is likely to provide a probiotic environment to assure fetal wellbeing. A stronger anti-inflammatory response (Noda-Nicolau et al., 2016) and the absence of IL-6 trans-signaling support this hypothesis. However, access of these microbes to fetal organs can contribute to adverse neonatal outcomes (Waites et al., 2005).

G. vaginalis increases the expression levels of IL-6 and the membrane-bound receptor proteins mIL-6R and gp130; however, gene expression of these receptors was downregulated. Considering that, the turnover times for proteins and mRNA of the same gene are not similar, i.e., the protein may stay longer than the mRNA(Eden et al., 2011; Yen et al., 2008), and the level of mRNA in steady state may be low, but protein translated can accumulate over time(Vogel and Marcotte, 2013). Moreover, since a protein is expressed from its corresponding mRNA, mRNA is prone to degradation. We firstly suggested that low concentration of mRNA and high mIL-6R protein occur because after translation, that particular mRNA gets degraded while its protein has higher half-life and remains in the cell. Secondly, considering that the expression is dynamics and we performed assays in one time point $(24 \mathrm{~h})$, this controversial result could be a transient state where functionally viable protein expression is already effective and could be identified, while mRNA of mIL-6R was not in steady state and could not be detected. This hypothesis becomes relevant since IL- 6 production in amnion cells is normally increased by $12 \mathrm{~h}$ of bacterial stimulation (Reisenberger et al., 1997). Lastly, since mRNA are regulated by several factors such as microRNAs(Wu and Brewer, 2012), we suggested that the decreased genic expression of mIL-6R could be a regulation mechanism, once there is mIL-6R protein sufficient in the tissue after $24 \mathrm{~h}$ and the inflammatory response needs to be regulated. Although the signal transducing chain gp130 is shared by members of the IL- 6 family of cytokines, mIL-6R is a unique binding receptor for IL-6 (Tanaka et al., 2014). Therefore, it is likely that the fetal membrane may try to minimize the induction of an intense inflammatory response by $G$. vaginalis (as seen by increased IL- 6 and all other associated signaling markers) by minimizing membrane receptors after $24 \mathrm{~h}$.

Other factors that could contribute to the function of IL-6 include IL6 and IL-6R polymorphisms and their interactions with infection, which can strongly influence IL-6 production and signaling (Velez et al., 2008). Further studies will be needed to determine how they may impact the host response to infection of the fetal membranes.

The observed IL- 6 increase by various PIAC-associated bacteria suggest its potential as a biomarker of infection, irrespective of the bacteria involved. However, an increase in this cytokine alone may not be indicative of any functional role, as its biological activities are controlled by additional molecules whose expression also changes in response to infection. IL- 6 change alone (increase or decrease) may not have functional relevance in fetal cells as shown in our study; however, we do not rule out the possibility of an impact of IL- 6 change in other tissues such as decidua, which is an extremely immunocompetent compartment. Additional information will be valuable to determine the possible downstream effects of increased IL-6 levels and/or to design an interventional target to modulate IL-6 function. As shown here, not all bacteria or combinations of them produce a molecular arrangement for IL-6 to exert a function. IL-6 expression by membranes is likely a nonspecific innate response and not a functional mediator of any laborinducing pathways. Indeed, previous studies have demonstrated that IL6 is neither necessary nor sufficient to include PTB in mouse models (Sadowsky et al., 2006; Yoshimura and Hirsch, 2003). Conversely, Robertson's group (Robertson et al., 2010) have shown that IL-6 null mutant mice have an extended gestational period after LPS treatment, suggesting yet another role for IL-6 in pregnancy maintenance.

\section{Conclusions}

Therefore, additional consideration of all the factors that influence IL- 6 bioactivity need to be considered if it is to be used as a biomarker for adverse pregnancy outcomes or a target for designing drugs to prevent them.

\section{Conflict of interest}

The authors report no conflict of interest.

\section{Funding}

This study was supported by: developmental funds granted to Dr. R Menon from the Department of OBGYN, the University of Texas Medical Branch; Galveston, TX, USA; by São Paulo Research Foundation - FAPESP (Grant 2012/17234-1) granted to Dr. Márcia Guimarães da Silva from the Department of Pathology, Botucatu Medical School, UNESP - Univ. Estadual Paulista, Botucatu, São Paulo, Brazil; and by Coordination for the Improvement of Higher Education Personnel - CAPES (BEX 3511/13-8) granted to Nathália Mayumi Noda Nicolau, PhD student in the Post-graduate Program in Pathology, Botucatu Medical School, UNESP - Univ. Estadual Paulista, Botucatu, São Paulo, Brazil. Mrs Nicolau performed her training at the University of Texas Medical Branch at Galveston (UTMB) with Dr. Menon.

\section{Acknowledgments}

None.

\section{References}

Aagaard, K., Ma, J., Antony, K., Ganu, R., Petrosino, J., Versalovic, J., 2014. The placenta harbors a unique microbiome. Sci. Transl. Med. 6, 1-22. http://dx.doi.org/10.1126/ scitranslmed.3008599.

Capoccia, R., Greub, G., Baud, D., 2013. Ureaplasma urealyticum, Mycoplasma hominis and adverse pregnancy outcomes. Curr. Opin. Infect. Dis. 26, 231-240. http://dx.doi. org/10.1097/QCO.0b013e328360db58.

Chaemsaithong, P., Romero, R., Korzeniewski, S.J., Martinez-Varea, A., Dong, Z., Yoon, B.H., Hassan, S.S., Chaiworapongsa, T., Yeo, L., 2015a. A point of care test for interleukin- 6 in amniotic fluid in preterm prelabor rupture of membranes: a step toward the early treatment of acute intra-amniotic inflammation/infection. J. Maternal-Fetal Neonatal Med. 7058, 1-8. http://dx.doi.org/10.3109/14767058. 2015.1006621.

Chaemsaithong, P., Romero, R., Korzeniewski, S.J., Martinez-Varea, A., Dong, Z., Yoon, B.H., Hassan, S.S., Chaiworapongsa, T., Yeo, L., 2015b. A rapid interleukin-6 bedside test for the identification of intra-amniotic inflammation in preterm labor with intact membranes. J. Maternal-Fetal Neonatal Med. 7058, 1-30. http://dx.doi.org/10. 3109/14767058.2015.1006620.

Choi, S.J., Park, S.D., Jang, I.H., Uh, Y., Lee, A., 2012. The prevalence of vaginal microorganisms in pregnant women with preterm labor and preterm birth. Ann. Lab. Med. 32, 194-200. http://dx.doi.org/10.3343/alm.2012.32.3.194.

DiGiulio, D.B., Romero, R., Kusanovic, J.P., Gómez, R., Kim, C.J., Seok, K.S., Gotsch, F., Mazaki-Tovi, S., Vaisbuch, E., Sanders, K., Bik, E.M., Chaiworapongsa, T., Oyarzún, E., Relman, D.a., 2010. Prevalence and diversity of microbes in the amniotic fluid, the fetal inflammatory response, and pregnancy outcome in women with preterm pre- 
labor rupture of membranes. Am. J. Reprod. Immunol. 64, 38-57. http://dx.doi.org/ 10.1111/j.1600-0897.2010.00830.x.

DiGiulio, D.B., 2012. Diversity of microbes in amniotic fluid. Semin. Fetal Neonatal Med. 17, 2-11. http://dx.doi.org/10.1016/j.siny.2011.10.001.

Doyle, R.M., Alber, D.G., Jones, H.E., Harris, K., Fitzgerald, F., Peebles, D., Klein, N., 2014. Term and preterm labour are associated with distinct microbial community structures in placental membranes which are independent of mode of delivery. Placenta 35, 1099-1101. http://dx.doi.org/10.1016/j.placenta.2014.10.007.

Eden, E., Geva-Zatorsky, N., Issaeva, I., Cohen, a., Dekel, E., Danon, T., Cohen, L., Mayo, a., Alon, U., 2011. Proteome half-life dynamics in living human cells. Science 331 (6018), 764-768. http://dx.doi.org/10.1126/science.1199784.

Flores-Herrera, H., García-López, G., Díaz, N.F., Molina-Hernández, a., Osorio-Caballero, M., Soriano-Becerril, D., Zaga-Clavellina, V., 2012. An experimental mixed bacterial infection induced differential secretion of proinflammatory cytokines (IL-1 $\beta$, TNF $\alpha$ ) and proMMP-9 in human fetal membranes. Placenta 33, 271-277. http://dx.doi.org/ 10.1016/j.placenta.2012.01.007.

Fortunato, S.J., Menon, R., Swan, K.F., L, T.W., 1994. Organ culture of amniochorionic membrane in vitro. Am. J. Reprod. Immunol. 32, 184-187.

Goldenberg, R.L., Hauth, J.C., Andrews, W.W., 2000. Intrauterine infection and preterm delivery. N. Engl. J. Med. 342, 1500-1507.

Govender, S., Theron, G.B., Odendaal, H.J., Chalkley, L.J., 2009. Prevalence of genital mycoplasmas, ureaplasmas and chlamydia in pregnancy. J. Obstetrics Gynaecology 29, 698-701. http://dx.doi.org/10.3109/01443610903184033.

Helmo, F.R., Alves, E.A.R., Moreira, R.A.D.A., Severino, V.O., Rocha, L.P., Monteiro, M.L.G.D.R., Reis, M.A., Dos Etchebehere, R.M., Machado, J.R., Corrêa, R.R.M., 2017. Intrauterine infection, immune system and premature birth. J. Maternal-Fetal Neonatal Med. 0, 1-7. http://dx.doi.org/10.1080/14767058.2017.1311318.

Jones, S.a., Scheller, J., Rose-john, S., 2011. Therapeutic strategies for the clinical blockade of IL-6/gp130 signaling. J. Clin. Invest. 121, 3375-3383. http://dx.doi.org/ 10.1172/JCI57158. (but).

Kacerovský, M., Pavlovský, M., Tosner, J., 2009. Preterm premature rupture of the membranes and genital mycoplasmas. Acta Medica (Hradec Kralove) 52, 117-120.

Kim, a., Lee, E.S., Shin, J.C., Kim, H.Y., 2013. Identification of biomarkers for preterm delivery in mid-trimester amniotic fluid. Placenta 34, 873-878. http://dx.doi.org/10. 1016/j.placenta.2013.06.306.

Kwak, D.-W., Hwang, H.-S., Kwon, J.-Y., Park, Y.-W., Kim, Y.-H., 2014. Co-infection with vaginal Ureaplasma urealyticum and Mycoplasma hominis increases adverse pregnancy outcomes in patients with preterm labor or preterm premature rupture of membranes. J. Maternal-Fetal Neonatal Med. 27, 333-337. http://dx.doi.org/10. 3109/14767058.2013.818124.

Lee, S.E., Romero, R., Kim, E.-C., Yoon, B.H., 2009. A high Nugent score but not a positive culture for genital mycoplasmas is a risk factor for spontaneous preterm birth. J. Maternal-Fetal. Neonatal Med. 22, 212-217. http://dx.doi.org/10.1080/ 14767050802616994.

Lee, S.Y., Buhimschi, I.a., Dulay, A.T., Ali, U.a., Zhao, G., Abdel-Razeq, S.S., Bahtiyar, M.O., Thung, S.F., Funai, E.F., Buhimschi, C.S., 2011. IL-6 trans-signaling system in intra-amniotic inflammation, preterm birth, and preterm premature rupture of the membranes. J. Immunol. 186, 3226-3236. http://dx.doi.org/10.4049/jimmunol. 1003587.

Marconi, C., De Andrade Ramos, B.R., Peraçoli, J.C., Donders, G.G.G., Da Silva, M.G., 2011. Amniotic fluid interleukin-1 beta and interleukin-6, but not interleukin-8 correlate with microbial invasion of the amniotic cavity in preterm labor. Am. J. Reprod. Immunol. 65, 549-556. http://dx.doi.org/10.1111/j.1600-0897.2010. 00940.x.

Menard, J.P., Mazouni, C., Salem-Cherif, I., Fenollar, F., Raoult, D., Boubli, L., Gamerre, M., Bretelle, F., 2010. High vaginal concentrations of Atopobium vaginae and Gardnerella vaginalis in women undergoing preterm labor. Obstetrics Gynecology 115, 134-140. http://dx.doi.org/10.1097/AOG.0b013e3181c391d7.

Mendz, G.L., Kaakoush, N.O., Quinlivan, J.a., 2013. Bacterial aetiological agents of intraamniotic infections and preterm birth in pregnant women. Front. Cell. Infect. Microbiol. 3, 58. http://dx.doi.org/10.3389/fcimb.2013.00058.

Menon, R., Williams, S.M., Fortunato, S.J., 2007. Amniotic fluid interleukin-1 $\beta$ and interleukin-8 concentrations: racial disparity in preterm birth. Reprod. Sci. 14, 253-259. http://dx.doi.org/10.1177/1933719107301336.

Menon, R., Peltier, M.R., Eckardt, J., Fortunato, S.J., 2009. Diversity in cytokine response to bacteria associated with preterm birth by fetal membranes. Am. J. Obstetrics Gynecology 201, 306.e1-306.e6. http://dx.doi.org/10.1016/j.ajog.2009.06.027.

Menon, R., Torloni, M.R., Voltolini, C., Torricelli, M., Merialdi, M., Betran, a.P., Widmer, M., Allen, T., Davydova, I., Khodjaeva, Z., Thorsen, P., Kacerovsky, M., Tambor, V., Massinen, T., Nace, J., Arora, C., 2011. Biomarkers of spontaneous preterm birth: an overview of the literature in the last four decades. Reprod. Sci. 18, 1046-1070. http://dx.doi.org/10.1177/1933719111415548.

Musilova, I., Bestvina, T., Hudeckova, M., Michalec, I., Cobo, T., Jacobsson, B., Kacerovsky, M., 2016. Vaginal fluid interleukin- 6 concentrations as a point-of-care test is of value in women with preterm prelabor rupture of membranes. Am. J. Obstetrics Gynecology 215, 619.e1-619.e12. http://dx.doi.org/10.1016/j.ajog.2016. 07.001 .
Naessens, A., Foulon, W., Breynaert, J., Lauwers, S., 1989. Postpartum bacteremia and placental colonization with genital mycoplasmas and pregnancy outcome. Am. J. Obstetrics Gynecology 160, 647-650.

Noda-Nicolau, N.M., Polettini, J., Peltier, M.R., da Silva, M.G., Menon, R., 2016. Combinations and loads of bacteria affect the cytokine production by fetal membranes: an in vitro study. Am. J. Reprod. Immunol. 76, 504-511. http://dx.doi.org/ 10.1111/aji.12596.

Reisenberger, K., Egarter, C., Schiebel, I., Obermair, A., Kiss, H., Lehner, R., 1997. In vitro cytokine and prostaglandin production by amnion cells in the presence of bacteria. Am. J. Obstetrics Gynecology 176, 981-984. http://dx.doi.org/10.1016/S00029378(97)70389-2.

Robertson, S.a., Christiaens, I., Dorian, C.L., Zaragoza, D.B., Care, A.S., Banks, A.M., Olson, D.M., 2010. Interleukin-6 is an essential determinant of on-time parturition in the mouse. Endocrinology 151, 3996-4006. http://dx.doi.org/10.1210/en.20100063.

Romero, R., Espinoza, J., Gonçalves, L.F., Kusanovic, J.P., Friel, L.a., Nien, J.K., 2006 Inflammation in preterm and term labour and delivery. Semin. Fetal Neonatal Med. 11, 317-326. http://dx.doi.org/10.1016/j.siny.2006.05.001.

Romero, R., Miranda, J., Chaiworapongsa, T., Chaemsaithong, P., Gotsch, F., Dong, Z., Ahmed, A.I., Yoon, B.H., Hassan, S.S., Kim, C.J., Korzeniewski, S.J., Yeo, L., 2014. A novel molecular microbiologic technique for the rapid diagnosis of microbial invasion of the amniotic cavity and intra-amniotic infection in preterm labor with intact membranes. Am. J. Reprod. Immunol. 71, 330-358. http://dx.doi.org/10.1111/aji. 12189.

Rose-John, S., 2012. Il-6 trans-signaling via the soluble IL-6 receptor: importance for the proinflammatory activities of IL-6. Int. J. Biol. Sci. 8, 1237-1247. http://dx.doi.org/ $10.7150 /$ ijbs. 4989.

Ryckman, K., Simhan, H., Krohn, M., Willians, S., 2009. Cervical cytokine network patterns during pregnancy: the role of bacterial vaginosis and geographic ancestry. J. Reprod. Immunol. 79, 174-182.

Sadowsky, D.W., Adams, K.M., Gravett, M.G., Witkin, S.S., Novy, M.J., 2006. Preterm labor is induced by intraamniotic infusions of interleukin-1 $\beta$ and tumor necrosis factor- $\alpha$ but not by interleukin- 6 or interleukin- 8 in a nonhuman primate model. Am J. Obstetrics Gynecology 195, 1578-1589. http://dx.doi.org/10.1016/j.ajog.2006. 06.072 .

Santos-Greatti, M.M.D.V., da Silva, M.G., Ferreira, C.S.T., Marconi, C., 2016. Cervicovaginal cytokines, sialidase activity and bacterial load in reproductive-aged women with intermediate vaginal flora. J. Reprod. Immunol. 118, 36-41. http://dx. doi.org/10.1016/j.jri.2016.08.005.

Shepard, M.C., Lunceford, C.D., 1967. Occurrence of urease in T strains of Mycoplasma. J. Bacteriol. 93, 1513-1520.

Steel, J.H., Malatos, S., Kennea, N., Edwards, a. D., Miles, L., Duggan, P., Reynolds, P.R., Feldman, R.G., Sullivan, M.H.F., 2005. Bacteria and inflammatory cells in fetal membranes do not always cause preterm labor. Pediatr. Res. 57, 404-411. http://dx. doi.org/10.1203/01.PDR.0000153869.96337.90.

Tanaka, T., Narazaki, M., Kishimoto, T., 2014. IL-6 in inflammation, immunity, and disease. Cold Spring Harb. Perspect. Biol. 6, a016295.

Taylor, B.D., Holzman, C.B., Fichorova, R.N., Tian, Y., Jones, N.M., Fu, W., Senagore, P.K., 2013. Inflammation biomarkers in vaginal fluid and preterm delivery. Hum. Reprod. 28, 942-952. http://dx.doi.org/10.1093/humrep/det019.

Velez, D.R., Fortunato, S.J., Williams, S.M., Menon, R., 2008. Interleukin-6 (IL-6) and receptor (IL6-R) gene haplotypes associate with amniotic fluid protein concentrations in preterm birth. Hum. Mol. Genet. 17, 1619-1630. http://dx.doi.org/10.1093/hmg/ ddn049.

Vogel, C., Marcotte, E.M., 2013. Insights into regulation of protein abundance from proteomics and transcriptomis analyses. Nat. Rev. Genet. 13, 227-232. http://dx.doi. org/10.1038/nrg3185. (Insights).

Waites, K.B., Katz, B., Schelonka, R.L., 2005. Mycoplasmas and ureaplasmas as neonatal pathogens. Clin. Microbiol. Rev. 18, 757-789. http://dx.doi.org/10.1128/CMR.18.4. 757-789.2005.

Wu, X., Brewer, G., 2012. The regulation of mRNA stability in mammalian cells: 2.0. Gene 500, 10-21. http://dx.doi.org/10.1016/j.gene.2012.03.021.

Yen, H.-C.S., Xu, Q., Chou, D.M., Zhao, Z., Elledge, S.J., 2008. Global protein stability profiling in mammalian cells. Science 322 (5903), 918-923. http://dx.doi.org/10 1126 /science. 1160489.

Yoon, B.H., Romero, R., Lim, J.-H., Shim, S.-S., Hong, J.-S., Shim, J.-Y., Jun, J.K., 2003. The clinical significance of detecting Ureaplasma urealyticum by the polymerase chain reaction in the amniotic fluid of patients with preterm labor. Am. J. Obstetrics Gynecology 189, 919-924.

Yoshimura, K., Hirsch, E., 2003. Interleukin-6 is neither necessary nor sufficient for preterm labor in a murine infection model. J. Soc. Gynecologic Invest. 10, 423-427. http://dx.doi.org/10.1016/S1071-55760300138-2.

Zaga-Clavellina, V., Garcia-Lopez, G., Flores-Herrera, H., Espejel-Nuñez, A., Flores-Pliego, A., Soriano-Becerril, D., Maida-Claros, R., Merchant-Larios, H., Vadillo-Ortega, F., 2007. In vitro secretion profiles of interleukin (IL)-1beta, IL-6, IL-8, IL-10, and TNF alpha after selective infection with Escherichia coli in human fetal membranes. Reprod. Biol. Endocrinol. 5, 46. http://dx.doi.org/10.1186/1477-7827-5-46. 